\title{
Let's Talk about the Association between Schizophrenia Polygenic Risk Scores and Cognition in Patients and the General Population: A Review
}

\author{
Sabrina K. Schaupp ${ }^{1,2}$, Thomas G. Schulze ${ }^{1}$, Monika Budde ${ }^{1, *}$ \\ ${ }^{1}$ Institute of Psychiatric Phenomics and Genomics (IPPG), University Hospital, \\ LMU Munich, Munich 80336, Germany; \\ ${ }^{2}$ Clinic for Psychiatry, Psychotherapy and Psychosomatics, Augsburg 86156, \\ Germany.
}

* Corresponding Author: Monika Budde. Email: monika.budde@med.uni-muenchen.de.

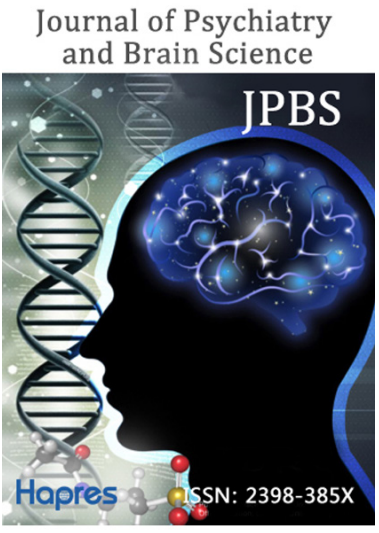

https://jpbs.hapres.com

\section{GOPEN ACCESS}

DOI: 10.20900/jpbs.20180012

Received: November 21, 2018

Accepted: December 13, 2018

Published: December 19, 2018

Copyright: $\odot 2018$ by the authors, Licensee Hapres, London, United Kingdom. This is an open access article distributed under the terms and conditions of the Creative Commons Attribution License (CC BY 4.0, https://creativecommons.org/licenses/by/4.0/).

\begin{abstract}
In the late 19th century, Emil Kraepelin divided endogenous psychosis into manic-depressive psychosis and dementia praecox. The latter term described individuals with schizophrenia and highlights cognitive deficits as a core feature of this illness. Since first degree relatives show lower cognitive performance than healthy controls, but better performance than patients, cognitive functions are discussed as potential endophenotypes for schizophrenia. During the last years, several studies have explored the relationship between schizophrenia polygenic risk scores and cognition both in individuals with psychiatric diseases and the general population. Current research shows mixed results for specific cognitive domains as well as general cognitive abilities and intelligence. These ambiguous results in parts might be due to the heterogeneity of neuropsychological tests used to measure various cognitive domains. Most studies are also underpowered, given the small to moderate effects of schizophrenia polygenic risk scores on cognition. As sufficient sample sizes become more and more available by international consortia and national registries, future studies will probably shed light on the biological relationship between schizophrenia and cognition.
\end{abstract}

Keywords: psychosis; genomic profile score; cognitive performance; intelligence; endophenotype 


\section{INTRODUCTION}

\section{Why consider schizophrenia polygenic risk scores when it comes to cognition?}

Since the very early stages of medical research on psychiatric disorders it has been evident that mental illnesses like bipolar disorder (BD), major depressive disorder (MDD), schizoaffective disorder (SA) or schizophrenia (SZ) are accompanied by cognitive deficits. Already the division of endogenous psychosis into manic-depressive psychosis and dementia praecox by Emil Kraepelin in the late 19th century emphasizes a continuous cognitive decline in patients who suffer from dementia praecox-today known as SZ. To this day, impairments in cognitive performance constitute one of the core features of $\mathrm{SZ}$ for a couple of reasons. First of all, cognitive impairments are present at all stages of the illness. Acute psychotic episodes are usually preceded by a prodromal phase. In this period, a loss of cognitive abilities is already present along with further unspecific symptoms like social withdrawal and affective flattening. In particular, cognitive performance serves as an important predictor in individuals who are at high risk for psychosis: high risk individuals who show more severe cognitive impairments are more likely to develop SZ in less than two years ${ }^{[1]}$. Furthermore, cognitive impairments not only remain present during acute psychotic episodes, but often last together with negative symptoms and are to this day hardly treatable. Interestingly, first degree relatives show lower cognitive performance than healthy controls, but better performance than patients ${ }^{[2]}$.

Cognitive impairments are most severe in SZ, but also occur in individuals who suffer from psychiatric disorders like MDD, BD and SA. However, the degree of cognitive impairments differs between the mentioned mental illnesses as well as their subtypes ${ }^{[3-7]}$. Besides this (endo-)phenotypic overlap, these disorders are genetically correlated ${ }^{[8]}$. Therefore, researchers have questioned the Kraepelinian dichotomy and proposed a continuum model from MDD and BD over $S A$ to $S Z^{[9]}$. A recent study investigated cognitive performance within a continuum approach, showing a decrease of cognitive abilities in the direction from $\mathrm{BD}$ to $\mathrm{SZ}{ }^{[10]}$

Due to the lack of a unique theory on cognition and its subdomains, measuring cognitive performance does not follow a homogenous path; rather the underlying theory on cognition and certain cognitive areas determines the choice of neurocognitive tests. Also, this heterogeneity is predominant in research on cognition in SZ, and amongst others a reason for the persisting ignorance on which domains are really affected in SZ. Therefore, a consortium of researchers developed the Measurement and Treatment Research to Improve Cognition in
Schizophrenia (MATRICS) Consensus Cognitive Battery (MCCB) ${ }^{[11]}$, to validly measure cognitive deficits in affected people. Furthermore, this battery should serve as a common instrument for clinical research on cognition with individuals who suffer from SZ to guarantee good replicability ${ }^{[11]}$. They identified seven important cognitive areas: attention/vigilance, working memory, verbal learning and memory, visual learning and memory, reasoning and problem solving, speed of processing, as well as social cognition (the latter is not part of this review). Accordingly, they defined neurocognitive tests for these areas ${ }^{[11]}$. The validity of the MCCB is supported by a study of August and colleagues ${ }^{[12]}$.

The observation that there is familial incidence of SZ led to a discussion on genetic causes for the disease. Twin and family studies showed evidence for the high heritability of SZ with estimates of up to $79 \%{ }^{[13]}$. Genome wide association studies (GWAS) have been promising for the identification of the underlying genetic mechanisms of SZ and revealed the highly polygenic architecture of this disease. The latest GWAS on SZ found 145 genome-wide significant loci ${ }^{[14]}$. Summary statistics from GWAS can be used to estimate the polygenic load of common risk alleles an individual carries for a certain trait or disorder in a polygenic risk score (PRS) ${ }^{[15,16]}$, in this case for $S Z$ (schizophrenia polygenic risk score, SZ-PRS).

However, as for most complex traits, the genetic cause of this disorder is still not fully understood. Therefore, so called endophenotypes for SZ are discussed, which might deliver a better reflection of the underlying genetic and biological risk factors. Cognitive deficits have first been described as a potential endophenotype for SZ by Gottesman and Shields in $1973^{[17]}$ and have been revised by Gottesman and Gould in $2003^{[18]}$. An endophenotype is (a) associated with the illness, (b) heritable, (c) independent of illness state, (d) co-segregates with illness in families, and as further suggestion (e) non-affected families have a higher rate than the general population ${ }^{[18]}$.

The association of cognitive impairments with SZ (criterion a) has been described above. Heritability estimates of intelligence (criterion b) in the general population range from $30-80 \%{ }^{[19]}$. Interestingly, despite high age-to-age genetic correlations the heritability of intelligence increases with age ${ }^{[20]}$. Thus, the same genetic variants affect intelligence at each stage in life, but the genetic variation between individuals accounts for more variance of the phenotype as age increases (for a detailed discussion of this topic, see references ${ }^{[20,21]}$ ). Recent GWAS of intelligence have revealed up to 205 significant loci, highlighting the importance of genetic pathways related to nervous system development, neuron differentiation as well as synapse structure and 
activity ${ }^{[22]}$. Similarly to findings from other complex traits like psychiatric disorders, these significant loci only explain up to $5.2 \%$ of the total heritability of intelligence ${ }^{[22]}$, which is an important indicator for a highly polygenic architecture. Furthermore, studies have reported a negative genetic correlation between general cognitive ability and $S Z^{[23,24]}$.

Bringing together knowledge on cognitive performance in SZ and its genetic basis, recent studies hypothesize that higher SZ-PRSs are associated with poorer cognitive performance both in patients and the general population. This review aims to present a differentiated overview of the current literature on this topic. For both parts, individuals with psychiatric diseases, predominantly SZ, and general population, results are presented in the same order, ranging from specific cognitive domains (attention/vigilance, working memory, verbal learning and memory, visual learning and memory, reasoning and problem solving, and speed of processing) to general cognitive abilities and intelligence. The latter two are highly correlated as they represent broad underlying cognitive abilities. Nevertheless they are presented separately in this review. As intelligence is reported as a standardized intelligence quotient (IQ) measure (although measured by different tests), general cognitive abilities are operationalized in most studies as a composite score of the available tests. Hence the outcome measure is highly dependent on the choice of tests and therefore not standardized to a comparable scale. A brief description of the cognitive domains discussed in this review is given in the INFOBOX.

\section{INFOBOX}

Attention/vigilance describes processes related to the perception of internal as well as external stimuli. Underlying processes are orientation (alertness, sustained attention/vigilance) towards and selection (selective attention and divided attention) of information.

Memory embraces all processes related to encoding, storage and recall of information. The classification of memory depends on duration (short-term memory, working memory, long-term memory) as well as explicit (semantic, episodic) and implicit content (procedural, priming). The working memory enables to temporarily store and manipulate information simultaneously. Encoding, storage and recall of information may occur on visual (visual learning and memory) or verbal (verbal learning and memory) material.

Executive functions are a regulation and control mechanism supporting a goal-oriented and situation specific behaviour, like planning, problem solving, and reasoning. Executive functions are activated as soon as automatic actions are not goal oriented anymore for problem solving.

Speed of processing is the time a person needs for a mental task, i.e., the time between receiving and responding to a stimulus. It is essential for all other cognitive skills.

General cognitive abilities are a heterogeneous measure. This construct embraces different cognitive skills depending on the administered tests without fulfilling the criteria for intelligence.

Intelligence embraces all cognitive performance parameters. There is no homogeneous description of intelligence. The most prominent theory is Spearman's two factor theory ( $\mathrm{g}$ - and s-factor) (for the interested reader, also consider Thurstone's model on primary mental abilities, Cattell's theory of fluid and crystalline intelligence). The g-factor is defined as an underlying general mental ability that influences the overall performance on different tasks.

\section{METHODS}

\section{Which studies were selected?}

Literature search was performed in the databases PubMed and Psychlnfo on July 26th, 2018 using the search terms "polygenic risk score schizophrenia" and "genomic risk profile score schizophrenia" combined with "cognition", "intelligence", "cognitive", "neuropsychology" and "neurocognitive", respectively. We screened the abstracts of the resulting articles and only considered studies with at least one objective measure of cognitive skills, i.e., a standardized test. Findings on soft measures of cognitive skills, for example parents' ratings on their children's ability to focus, are not reported in this review. In addition, we hand-searched the references of the selected articles and reviews for further suitable publications.

In studies comparing cases and controls, exclusion criteria often apply for the control group like having a psychiatric diagnosis or having affected 
relatives. In contrast, these exclusion criteria do not typically apply in studies working with samples from the general population. Furthermore, there are important confounders of cognitive performance in a clinical sample that occur less frequently in samples from the general population like medication, drug abuse, psychotic symptoms and age at onset. Therefore, we decided to review these two types of studies separately.

\section{RESULTS}

\subsection{Is there an association between schizophrenia polygenic risk scores} (SZ-PRS) and cognitive performance in individuals with psychiatric diseases?

All studies performed in individuals with psychiatric diseases are listed in Table 1. The studies reviewed for this article did not administer the Measurement and Treatment Research to Improve Cognition in
Schizophrenia (MATRICS) Consensus Cognitive Battery (MCCB) in its full extent, but in summary most of them used at least certain tests which are defined in the MCCB (Table 1). In total our search delivered ten studies (for detailed information on the respective samples see Table 1). SZPRS differentiated significantly between cases, relatives and controls, as well as within cases between different diagnoses (schizophrenia (SZ), bipolar disorder (BD), major depressive disorder (MDD), schizoaffective disorder (SA)), with the highest loading in individuals with SZ. Consistent with other studies ${ }^{[25]}$, only a small part of disease status could be explained by SZ-PRS. Four of the reviewed studies considered intelligence as cognitive measure. The other six studies differ from each other in their approach. While some consider a composite outcome (general cognitive abilities) based on different neurocognitive tests for at least twocognitive domains, others investigated single cognitive areas or combined both approaches. These inconsistencies in approaches used also reflect the heterogeneity in results found.

Table 1. Studies on SZ-PRS and cognition in patients, relatives and controls.

\begin{tabular}{|c|c|c|}
\hline Author \& Year & Sample & Domain (Test) *Significant \\
\hline Alloza et al., 2017 ${ }^{[26]}$ & $\begin{array}{l}\text { - C: } N=36 \\
\text { - P: SZ: } N=28\end{array}$ & $\begin{array}{l}\text { - IQ (vocabulary, block design, } \\
\text { similarities (WAIS)) }\end{array}$ \\
\hline $\begin{array}{l}\text { Nakahara et al., } \\
2018^{[27]}\end{array}$ & $\begin{array}{l}\text { - C: } N=136 \\
\text { - P: } S Z: N=127\end{array}$ & $\begin{array}{l}\text { - speed of processing * (CMINDS) } \\
\text { - attention/vigilance * (CMINDS) } \\
\text { - working memory * (CMINDS) } \\
\text { - verbal memory * (CMINDS) } \\
\text { - visual memory * (CMINDS) } \\
\text { - reasoning/problem solving * (CMINDS) } \\
\text { - general cognitive abilities * (CMINDS) }\end{array}$ \\
\hline $\begin{array}{l}\text { Ranlund et al., } \\
2017^{[28]}\end{array}$ & $\begin{array}{l}\text { - C: MDD: } N=137 ; \\
\text { AD: } N=15 ; \\
\text { other non-psychotic disorder: } N=20 \text {; } \\
\text { no psychiatric illness = } 2161 \\
\text { R: MDD: } N=136 ; \\
\text { AD: } N=32 ; \\
\text { other non-psychotic disorder: } N=21 \text {; } \\
\text { no psychiatric disorder: } N=633 \\
\text { - P: SZ: } N=703 ; \\
\text { - BD: } N=105 ; \\
\text { - SA: } N=60 ; \\
\text { other psychotic disorder: } N=219\end{array}$ & $\begin{array}{l}\text { - attention \& working memory } \\
\text { (forward and backward digit span) } \\
\text { - spatial visualization * } \\
\text { (block design) } \\
\text { - short- and long-term verbal memory } \\
\text { (RAVLT) }\end{array}$ \\
\hline $\begin{array}{l}\text { Shafee et al., } \\
2018^{[29]}\end{array}$ & $\begin{array}{l}\text { Bipolar-Schizophrenia Network for } \\
\text { Intermediate Phenotypes: } \\
\text { - C: } N=180 \text { (no history of psychosis) } \\
\text { - R: } N=243 \text { (no history of psychosis) } \\
\text { - P: SZ: } N=100 ; \text { BD: } N=143 ; \text { SA: } N=71 \\
\text { - Philadelphia Neurodevelopmental } \\
\text { - Cohort: C: } N=4511\end{array}$ & $\begin{array}{l}\text { - general cognitive abilities (BACS) } \\
\text { - IQ (WRAT) }\end{array}$ \\
\hline
\end{tabular}


Table 1. Cont.

\begin{tabular}{|c|c|c|}
\hline Author \& Year & Sample & Domain (Test) *Significant \\
\hline $\begin{array}{l}\text { van Os et al., } \\
2017^{[30]}\end{array}$ & $\begin{array}{l}\text { Genetic Risk and Outcome of Psychosis: } \\
\text { - C: } N=586 \text { (other non-psychotic } \\
\text { disorder: } N=59 \text { ) } \\
\text { - R: siblings of P: } N=1059 \text { (other } \\
\text { non-psychotic disorder: } N=154 \text { ); } \\
\text { parents of } \mathrm{P}: N=920\end{array}$ & $\begin{array}{l}\text { - IQ (information, block design, digit } \\
\text { symbol coding, arithmetic (WAIS); } \\
\text { baseline \& 3-year follow-up) } \\
\text { - IQ (information (every third item), block } \\
\text { design (uneven items), digit symbol } \\
\text { coding, arithmetic (uneven items) } \\
\text { (WAIS-III short form); 6-year follow-up) }\end{array}$ \\
\hline $\begin{array}{l}\text { Terwisscha van } \\
\text { cheltinga et al., } \\
2013^{[31]}\end{array}$ & $\begin{array}{l}\text { - C: } N=322 \\
\text { - P: SZ: } N=315 ; \text { SA: } N=35\end{array}$ & $\bullet$ IQ (WAIS III \& IIIR) \\
\hline $\begin{array}{l}\text { Walton et al., } \\
2013^{[32]}\end{array}$ & $\begin{array}{l}\text { - C: } N=99 \\
\text { - P: SZ: } N=79\end{array}$ & $\begin{array}{l}\text { - working memory (Sternberg Item } \\
\text { Recognition Paradigm) }\end{array}$ \\
\hline $\begin{array}{l}\text { Wang et al., } \\
2018^{[33]}\end{array}$ & $\begin{array}{l}\text { Schizophrenia Trio Genomic Research } \\
\text { in Taiwan: } \\
\text { - P: } N=1120\end{array}$ & $\begin{array}{l}\text { - sustained attention (CPT) } \\
\text { - executive function (WCST) } \\
\text { - general cognitive abilities * } \\
\text { (based on CPT \& WCST) }\end{array}$ \\
\hline $\begin{array}{l}\text { Whalley et al., } \\
2016^{[34]}\end{array}$ & $\begin{array}{l}\text { Generation Scotland:the Scottish } \\
\text { Family Health Study: } \\
\text { - C: } N=16764 \\
\text { - P: MDD: } N=2587 \\
\text { UK Biobank (Replication Sample): } \\
\text { - C: } N=27476 \\
\text { - P: MDD: } N=6049\end{array}$ & $\begin{array}{l}\text { - verbal declarative memory } \\
\text { (Wechsler Logical Memory III) } \\
\text { - processing speed (Digit Symbol } \\
\text { Coding (WAIS)) } \\
\text { - verbal ability (Mill Hill Vocabulary } \\
\text { Scale) } \\
\text { - executive function (letter-based } \\
\text { phonemic verbal fluency test) }\end{array}$ \\
\hline $\begin{array}{l}\text { Xavier et al., } \\
2018^{[35]}\end{array}$ & $\begin{array}{l}\text { Clinical Antipsychotics } \\
\text { Trials of Intervention } \\
\text { Effectiveness-schizophrenia trial: } \\
\text { - P: } N=741\end{array}$ & $\begin{array}{l}\text { - general cognitive abilities } \\
\text { (computerized test of visuo-spatial } \\
\text { working memory, HVLT, CPT, } \\
\text { COWAT, WCST) }\end{array}$ \\
\hline
\end{tabular}

* significant association (at least one $p$-value threshold); direction of significant effects: higher SZ-PRS were associated with poorer performance in the respective tests; $\mathrm{P}=$ individuals with psychiatric diseases; $\mathrm{R}=$ relatives; $\mathrm{C}=\mathrm{controls}$; $\mathrm{SZ}$ = schizophrenia; $\mathrm{BD}=$ bipolar disorder; $\mathrm{SA}=$ schizoaffective disorder; $\mathrm{MDD}=$ major depressive disorder; $\mathrm{AD}=$ anxiety disorder; WAIS = Wechsler Adult Intelligence Scale; CMINDS = Computerized Multiphasic Interactive Neurocognitive System; RAVLT = Rey Auditory Verbal Learning Test; BACS = Brief Assessment of Cognition in Schizophrenia; WRAT = Wide Range Achievement Test; CPT = continuous performance test; WCST = Wisconsin Card Sorting Test; HVLT = Hopkins Verbal Learning Test; COWAT = Controlled Oral Word Association Test.

\subsubsection{Attention/vigilance}

Since attention is strongly impaired in individuals with SZ, most studies considered this domain in their analyses. Significant associations between SZ-PRS and attention are only reported by Nakahara et al. ${ }^{[27]}$, while other studies did not reveal any significant association between SZ-PRS and attention ${ }^{[28,33]}$. Post hoc analysis by Xavier et al. ${ }^{[35]}$ revealed a significant association between SZ-PRS and vigilance but the finding did not survive correction for multiple testing.

\subsubsection{Working memory}

In the functional magnetic resonance imaging (fMRI) study by Walton et al. a significant association was shown between SZ-PRS and the dorsolateral prefrontal cortex, which is related to working memory ${ }^{[32]}$. However, this association could not be shown for the outcome measure of the administered test ${ }^{[32]}$. Similarly, Xavier et al. was not able to show a significant association between SZ-PRS and working memory in his post hoc analysis ${ }^{[35]}$. But based on the CMINDS a 
significant association between these two variables has been shown by Nakahara et al. ${ }^{[27]}$.

\subsubsection{Verbal learning and memory}

While the studies of Ranlund and Whalley ${ }^{[28,34]}$ were not able to find any association between SZ-PRS and verbal learning, Nakahara et al. ${ }^{[27]}$ showed a significant association between verbal learning and SZ-PRS. Also, in this case the post hoc analysis by Xavier et al. ${ }^{[35]}$ on a possible association between SZ-PRS and memory did not survive correction for multiple testing.

\subsubsection{Visual learning and memory}

Only the study of Nakaraha et al. ${ }^{[27]}$ considered visual learning and was able to show a significant association with SZ-PRS.

\subsubsection{Reasoning and problem solving}

The Wisconsin Card Sorting Test (WCST) is also part of the MCCB as a valid measurement for reasoning and problem solving as subfunctions of executive functioning and has been applied in the study of Wang et al. ${ }^{[33]}$. They report a significant association between the outcome measures perseverative responses, perseverative errors, conceptual level response and failure to maintain set and SZ-PRS, even though the overall outcome measure for the WCST did not reveal any significant association with SZ-PRS ${ }^{[33]}$. For problem solving Nakahara et al. ${ }^{[27]}$ report a significant negative correlation with SZ-PRS. Post hoc analysis by Xavier et al. ${ }^{[35]}$ did not reveal a significant association between SZ-PRS and reasoning.

\subsubsection{Speed of processing}

Nakahara et al. ${ }^{[27]}$ as well as Whalley et al. ${ }^{[34]}$ describe a significant association between SZ-PRS and speed of processing. However, there is a peculiarity in the study of Whalley et al. ${ }^{[34]}$. Their case sample refers to individuals with MDD. Another caveat is that they only report this significant association in one of the two analyzed samples ${ }^{[34]}$. The post hoc analysis by Xavier et al. ${ }^{[35]}$ did not reveal a significant association between SZ-PRS and speed of processing.

\subsubsection{General cognitive abilities}

Four out of ten studies considered a score for general cognitive abilities to investigate its association with SZ-PRS. This composite score however, must be interpreted with caution since each study investigated different cognitive areas and applied different neurocognitive tests, leading to inconsistent findings. Wang et al. ${ }^{[33]}$ based their outcome measure for general cognitive abilities on the neurocognitive tests for sustained attention (CPT) and executive functioning (WCST; see Table 1) and reported a significant association with SZ-PRS. The composite score of the CMINDS, applied by Nakahara et al. ${ }^{[27]}$, also showed a significant negative correlation between SZ-PRS and general cognitive abilities. Further reviewed studies have not been able to show a significant association between SZ-PRS and general cognitive abilities in cases ${ }^{[29,35]}$. Interestingly, Shafee et al. ${ }^{[29]}$ found a significant association between general cognitive abilities and SZ-PRS in the non-psychiatric group. Therefore, they assumed that cognitive decrease in patients was due to morbid factors like disease progression, protective effects, treatment effects and possible substance abuse ${ }^{[29]}$.

\subsubsection{Intelligence}

Three out of ten studies with cases and controls ${ }^{[26,29,31]}$ and one longitudinal study with relatives and controls ${ }^{[30]}$ analyzed a possible association between intelligence quotient (IQ) and SZ-PRS. Three out of four studies reported a significant difference in IQ with a lower IQ in individuals with psychiatric diseases ${ }^{[26,29,31]}$. None of the reviewed studies showed a significant association with SZ-PRS. However, one study found a nominally significant association ${ }^{[31]}$ or a trend toward a significant association of SZ-PRS with $I Q^{[26]}$, respectively. Further analysis of Terwisscha van Scheltinga et al. ${ }^{[31]}$ considered a possible association of SZ-PRS with specific cognitive functions, which also did not reveal any relevant results. They discussed that lack of association between SZ-PRS and IQ that both were independently associated with SZ. Besides, like Shafee et al. ${ }^{[29]}$ they argued that cognitive deficits might be due to secondary processes like SZ gene effects on brain growth and maturation as well as environmental interaction effects. The longitudinal study of van Os et al. ${ }^{[30]}$ with a follow-up after three and six years did not reveal a significant association between SZ-PRS and IQ in the group of relatives, while there was a significant association in the control group.

\subsection{Is there an association between SZ-PRS and cognitive performance in the general population?}

A total of twelve studies were considered (Table 2), eight of which explored the effects of SZ-PRS on specific cognitive domains. Overall, there were only a few significant findings. 
Table 2. Studies on SZ-PRS and cognition in the general population.

\begin{tabular}{|c|c|c|}
\hline Author \& Year & Sample & Domain (Test) *Significant \\
\hline $\begin{array}{l}\text { Benca et al., } \\
2017^{[36]}\end{array}$ & $\begin{array}{l}\text { Colorado Longitudinal Twin study (LTS) } \\
\text { and the Colorado Community Twin } \\
\text { Study (CTS) (mean age: } 19.6 \text { years): } \\
N=386\end{array}$ & $\begin{array}{l}\text { - executive functioning (antisaccade, } \\
\text { stop-signal, Stroop, keep track, letter } \\
\text { memory, spatial } n \text {-back, number-letter, } \\
\text { color-shape, category-switch) } \\
\text { - IQ (WAIS-III (LTS)/WASI (CTS)) }\end{array}$ \\
\hline $\begin{array}{l}\text { Córdova-Palomera } \\
\text { et al., } 2018^{[37]}\end{array}$ & $\begin{array}{l}\text { Philadelphia Neurodevelopmental } \\
\text { Cohort: } \\
N=4183 \text { (8-22 years) }\end{array}$ & - IQ * ,\# (WRAT) \\
\hline $\begin{array}{l}\text { Germine et al., } \\
2016^{[38]}\end{array}$ & $\begin{array}{l}\text { Philadelphia Neurodevelopmental } \\
\text { Cohort (Discovery sample; 8-21 years): } \\
N=4303 \\
\text { Harvard/Massachusetts General } \\
\text { Hospital Brain Genomics Superstruct } \\
\text { Project (Replication sample; 18-35 } \\
\text { years): } \\
N=695\end{array}$ & $\begin{array}{l}\text { Computerized Neurocognitive Battery: } \\
\text { - abstraction/cognitive flexibility } \\
\text { (Penn conditional exclusion test) } \\
\text { - attention (Penn continuous } \\
\text { performance test) } \\
\text { - working memory (letter n-back task) } \\
\text { - verbal memory (Penn word memory task) } \\
\text { - face memory (Penn face memory task) } \\
\text { - spatial memory (visual object learning test) } \\
\text { - verbal reasoning *(Penn verbal } \\
\text { reasoning test) } \\
\text { - nonverbal reasoning (Penn matrix } \\
\text { reasoning test) } \\
\text { - spatial reasoning (Penn line } \\
\text { orientation test) } \\
\text { - emotion identification * (Penn emotion } \\
\text { identification test (+replication sample)) } \\
\text { - emotion discrimination (Penn emotion } \\
\text { differentiation test) } \\
\text { - age discrimination (Penn age } \\
\text { differentiation test) } \\
\text { - motor speed (computerized finger } \\
\text { tapping test) } \\
\text { - gensorimotor speed (mouse practice task) } \\
\text { all tests) }\end{array}$ \\
\hline $\begin{array}{l}\text { Hagenaars et al., } \\
2016^{[39]}\end{array}$ & $\begin{array}{l}\text { UK Biobank Study: (40-73 years) } \\
-N=111484 \text { (reaction time) } \\
\text { - } N=112067 \text { (memory) } \\
\text { - } N=36035 \text { (verbal-numerical } \\
\text { reasoning) }\end{array}$ & $\begin{array}{l}\text { - reaction time * (computerized } \\
\text { "Snap" game) } \\
\text { - memory * (recalling cards) } \\
\text { - executive functioning * (verbal-numerical } \\
\text { reasoning task) }\end{array}$ \\
\hline $\begin{array}{l}\text { Hatzimanolis et al., } \\
2015^{[40]}\end{array}$ & $\begin{array}{l}\text { Athens Study of Psychosis Proneness } \\
\text { and Incidence of Schizophrenia } \\
\text { (Discovery sample; 18-24 years): } \\
\text { - } N=1079 \\
\text { Athens Study of Psychosis Proneness } \\
\text { and Incidence of Schizophrenia } \\
\text { (Replication sample): } \\
\text { - } N=738 \\
\text { Learning on Genetics of Schizophrenia } \\
\text { Spectrum (Replication sample; } \\
18-29 \text { years): } \\
\text { - } N=825\end{array}$ & $\begin{array}{l}\text { - } \text { non-verbal IQ (Raven's matrices) } \\
\text { - sustained attention/ vigilance (continuous } \\
\text { performance test, Identical Pairs version) } \\
\text { - verbal working memory (verbal } n \text {-back } \\
\text { (2-back)) } \\
\text { - spatial working memory (spatial } n \text {-back } \\
\text { (2-back)) } \\
\text { - oculomotor functioning (antisaccade eye } \\
\text { movements; smooth pursuit eye movements) }\end{array}$ \\
\hline
\end{tabular}


Table 2. Cont.

\begin{tabular}{|c|c|c|}
\hline Author \& Year & Sample & Domain (Test) *Significant \\
\hline $\begin{array}{l}\text { Hubbard et al., } \\
2015^{[23]}\end{array}$ & $\begin{array}{l}\text { Avon Longitudinal Study of Parents and } \\
\text { Children (8 years): } \\
\bullet N=5109-5556\end{array}$ & $\begin{array}{l}\text { - } \text { attention * (sky search task (TEA-Ch)) } \\
\text { - problem solving (block design (WISC-III)) } \\
\text { - processing speed (coding (WISC-III) } \\
\text { - social cognition (diagnostic analysis of } \\
\text { nonverbal accuracy) } \\
\text { - verbal learning *,\# (nonword } \\
\text { repetition test) } \\
\text { - working memory (digit span backward } \\
\text { (WISC-III) } \\
\text { - performance IQ * (WISC-III) } \\
\text { - verbal IQ (WISC-III) } \\
\text { - total IQ * (WISC-III) }\end{array}$ \\
\hline $\begin{array}{l}\text { Krapohl et al., } \\
2016^{[41]}\end{array}$ & $\begin{array}{l}\text { Twins Early Development Study (16 years): } \\
\bullet N=3152\end{array}$ & $\begin{array}{l}\text { - general cognitive abilities (Raven's } \\
\text { matrices, Mill Hill Vocabulary) } \\
\text { - non-verbal IQ (Raven's matrices) } \\
\text { - verbal IQ (Mill Hill Vocabulary) }\end{array}$ \\
\hline $\begin{array}{l}\text { Lencz et al., } \\
2014^{[42]}\end{array}$ & $\begin{array}{l}\text { COGENT: } \\
\bullet N=4896 \\
\text { (from nine samples; range of mean age } \\
\text { in samples: } 15.9-69.5 \text { years) }\end{array}$ & - general cognitive abilities * \\
\hline $\begin{array}{l}\text { Liebers et al., } \\
2016^{[43]}\end{array}$ & $\begin{array}{l}\text { Health and Retirement Study: } \\
\bullet N=8616\end{array}$ & $\begin{array}{l}\text { - general cognitive abilities * }(\text { TICS }) \\
\text { - attention/ orientation * (TICS) } \\
\text { - verbal memory * (TICS) }\end{array}$ \\
\hline $\begin{array}{l}\text { Mclntosh et al., } \\
2013^{[44]}\end{array}$ & $\begin{array}{l}\text { Lothian Birth Cohort } 1936 \text { (Discovery } \\
\text { sample; } T_{1}: 11 \text { years, } T_{2}: 70 \text { years): } \\
\text { - } N=937 \\
\text { Lothian Birth Cohort } 1921 \text { (Replication } \\
\text { sample; } T_{1}: 11 \text { years, } T_{2}: 79 \text { years): } \\
\text { - } N=517\end{array}$ & $\begin{array}{l}\text { - IQ (Moray House Test; discovery sample; } \\
\text { age } 11 \text { and 70) } \\
\text { - fluid-type general cognitive functioning * } \\
\text { (WAIS-III, WMS-III; discovery sample; age 70) } \\
\text { - general cognitive ability * (Moray House } \\
\text { Test, Raven's matrices, verbal fluency test, } \\
\text { logical memory test; replication sample; } \\
\text { age 79) }\end{array}$ \\
\hline $\begin{array}{l}\text { Rampino et al., } \\
2017^{[45]}\end{array}$ & $\begin{array}{l}\text { Discovery sample: } \\
\bullet N=260 \text { (mean age: } 28 \text { years) } \\
\text { Replication sample: } \\
-N=73 \text { (age: } 26 \text { years) }\end{array}$ & $\begin{array}{l}\text { - attention (continuous performance test) } \\
\text { - working memory ( } N \text {-Back test) } \\
\text { - speed of processing (Trail Making Test A, } \\
\text { B and B-A) }\end{array}$ \\
\hline $\begin{array}{l}\text { Riglin et al., } \\
2017^{[46]}\end{array}$ & $\begin{array}{l}\text { Avon Longitudinal Study of Parents and } \\
\text { Children (4-9 years): } \\
-N=5100-6952\end{array}$ & $\begin{array}{l}\text { - reading ability (Wechsler Objective } \\
\text { Reading Dimensions) } \\
\text { - verbal and performance * IQ (WISC) }\end{array}$ \\
\hline
\end{tabular}

*significant association (at least one p-value threshold); \#positive association, thus higher SZ-PRS associated with higher cognitive performance; direction of all other significant effects: higher SZ-PRS were associated with poorer performance in the respective tests; WISC = Wechsler Intelligence Scale for Children; WAIS = Wechsler Adult Intelligence Scale; WMS = Wechsler Memory Scale; TICS = Telephone Interview for Cognitive Status; TEA-Ch = Test of Everyday Attention for Children; WRAT = Wide Range Achievement Test. 


\subsubsection{Attention/vigilance}

Of the five studies analyzing measures of attention (see Table 2), only two found a significant association ${ }^{[23,43]}$. However, Hubbard et al. only reported significant results for SZ-PRS based on a genome wide association study (GWAS) in the Psychiatric Genomics Consortium (PGC) sample ${ }^{[25]}$, not in an additional sample of individuals with a clinical diagnosis of SZ (attending a clozapine clinic in the UK; CLOZUK sample ${ }^{[23]}$. Higher SZ-PRSs were associated with lower performance in attention tasks in the Telephone Interview for Cognitive Status ${ }^{[43]}$. It is important to note, that this was the study with the biggest sample size. One of the other studies reported nominally significant results ${ }^{[40]}$.

\subsubsection{Working memory}

None of the five studies reporting measures of working memory ${ }^{[23,36,38,40,45]}$ did find a significant effect of SZ-PRS, probably due to a lack of power.

\subsubsection{Verbal learning and memory}

Verbal learning and memory were significantly associated with SZ-PRS in two studies ${ }^{[23,43]}$. It is noteworthy, that Hubbard and colleagues did find a positive association, opposite to the hypothesized direction of effect ${ }^{[23]}$. Thus, higher SZ-PRS were related to a better performance in verbal learning. However, as for attention, they only reported significant results for SZ-PRS based on a GWAS in the PGC sample ${ }^{[25]}$, not in the CLOZUK sample ${ }^{[23]}$.

\subsubsection{Visual learning and memory}

Only two studies explored visual learning and memory. While Germine et al. did not see a significant association with SZ-PRS ${ }^{[38]}$, Hagenaars and colleagues reported a negative effect ${ }^{[39]}$. This inconsistency can most likely be explained by the huge difference regarding sample size between these studies.

\subsubsection{Reasoning and problem solving}

Measures of reasoning and problem solving were analyzed in three studies ${ }^{[23,38,39]}$, two of which report a significant negative association between SZ-PRS and verbal reasoning ${ }^{[38]}$ and verbal-numerical reasoning ${ }^{[39]}$ in the general population. Unlike verbal reasoning, nonverbal and spatial reasoning were not associated with SZ-PRS in the study by Germine and colleagues ${ }^{[38]}$.

\subsubsection{Speed of processing}

Speed of processing was measured in four studies (see Table 2). Similar to the other domains, only the study with the outstanding sample size was able to find a significant negative association with SZ-PRS ${ }^{[39]}$.

\subsubsection{General cognitive abilities}

As already discussed for the case-control studies, the estimates of the general cognitive abilities highly depend on the tests used to create the respective scores. The results are mixed. While two studies did not report significant results ${ }^{[38,41]}$, others did find a negative association between SZ-PRS and their estimates of general cognitive abilities ${ }^{[42-44]}$. In the study by Lencz and colleagues ${ }^{[42]}$, the negative association between SZ-PRS and general cognitive abilities was complementary to their main analysis showing that individuals with SZ had a significantly lower polygenic score for cognition than controls in different SZ-cohorts, highlighting the genetic overlap between these two traits. Regarding possible effects of SZ-PRS on cognitive changes with age, McIntosh and colleagues observed higher SZ-PRS associated with greater relative decline in general cognitive functioning between age 11 and age $70^{[44]}$. However, a longitudinal study of cognitive decline in older age did not find any effect of SZ-PRS ${ }^{[43]}$.

\subsubsection{Intelligence}

We found seven studies exploring possible associations between SZ-PRS and measures of IQ. The first one was published in 2013 and was the only one using SZ-PRS based on the PGC1 GWAS ${ }^{[15]}$. All other studies based the SZ-PRS on the summary statistics from the PGC2 GWAS ${ }^{[25]}$, in one case also on a GWAS in the CLOZUK sample ${ }^{[23]}$. This limited number of studies does not yet paint a clear picture. However, some interesting preliminary trends can be discussed. Three of the studies reported significant findings on some of the measures they used. Performance IQ showed more potential for associations with SZ-PRS than verbal $I^{[23,46]}$. Two studies found effects of SZ-PRS on general IQ measures ${ }^{[23,37]}$, while others did not ${ }^{[36,44]}$. In two studies, a higher SZ-PRS was associated with a lower IQ. However, Córdova et al. found an effect in the opposite direction ${ }^{[37]}$. This finding underlines the fact that the direction of the relationship between alleles associated with risk for SZ and cognition does not seem to be as clear as expected and is in line with studies showing a positive relationship between SZ-PRS and creativity ${ }^{[47]}$ and mixed results on the direction of the genetic correlation between $\mathrm{SZ}$ and educational attainment (positive correlation: ${ }^{[48]}$; negative correlation: ${ }^{[49]}$ ).

The decline in cognitive performance in individuals with SZ often starts in childhood or adolescence, 
preceding the first acute illness episode for years. Therefore, children and adolescents are important age groups to study the effects of SZ-PRS on cognition, which is reflected in the current literature. Five out of the six studies worked with samples including-albeit not necessarily exclusivelychildren and adolescents ${ }^{[23,37,41,44,46]}$, three of which found significant associations with SZ-PRS ${ }^{[23,37,46]}$. Intelligence is less heritable in childhood compared to adulthood ${ }^{[20]}$. Also, Hill and colleagues ${ }^{[50]}$ studied the age dependent pleiotropy between general cognitive function and major psychiatric disorders and found a negative genetic correlation between cognitive function and SZ in older age, but not in childhood. Even if not reflected in studies on SZ-PRS and cognition yet, as Mclntosh and colleagues $^{[44]}$ did not see a significant association of SZ-PRS and IQ at both age 11 and age 70, these age-dependent differences need to be considered when interpreting studies on genetic overlap between $\mathrm{SZ}$ and cognition.

\section{Discussion}

\subsection{What have we learned so far?}

As the concept of polygenic risk scores (PRS) has only been around for a decade, the number of studies investigating the relationship between schizophrenia PRS (SZ-PRS) and cognition is limited.

In summary, most studies with affected individuals did not find an association between SZ-PRS and cognition i.e., general cognitive abilities, specific cognitive domains or intelligence quotient (IQ). Only a few were able to find an association for general cognitive abilities ${ }^{[27,33]}$ and for specific cognitive domains ${ }^{[27,33]}$. The picture from studies in the general population is similarly blurry. Measures of IQ or general cognitive abilities vary a lot, which might in part explain the heterogeneous results. The field needs to balance the respective advantages and disadvantages of huge samples with comparatively broad and unspecific measures of cognition versus deeply phenotyped smaller samples. In general, the effect sizes of SZ-PRS on cognition are small to moderate. Most studies that found significant associations between SZ-PRS and intelligence or general cognitive abilities in the general population reported changes in $R^{2}$ due to SZ-PRS of $0.3 \%{ }^{[46]}$ at most (with the exception of Lencz et al., who saw an $R^{2}$ of $1.61 \%$ in one of their samples ${ }^{[42]}$. Thus, the amount of variance of the phenotype cognition explained by the genetic load of SZ risk loci is rather low. In contrast, the amount of genetic variance of cognition explained by the genetic load of SZ risk loci is higher. The Brainstorm Consortium recently reported a genetic correlation $\left(r_{\mathrm{g}}\right)$ of -0.19 between SZ and intelligence ${ }^{[51]}$, which would correspond to an $R^{2}$ of $3.61 \%$. Thus, between cognition and SZ, there seems to exist significant overlap of genetic factors $\left(r_{\mathrm{g}}\right)$, but SZ-PRS appear to influence the phenotype cognition to a much smaller degree. Therefore, big samples are needed to achieve the statistical power that is necessary to detect these effects, as is clearly reflected in the results. The findings from the reported studies suggest aiming for large samples (e.g., $N>110,000$ in $^{[39]}$ ) rather than very precisely measured cognitive domains in smaller samples ${ }^{[36]}$. Only with sufficient samples valid conclusions can be drawn.

Since there is an (endo-)phenotypic as well as genotypic overlap between bipolar disorder (BD), major depressive disorder (MDD), schizoaffective disorder (SA) and schizophrenia (SZ), some studies used mixed samples in their analyses ${ }^{[28,29,31,34]}$. In all studies SZ-PRS significantly differentiated between the different diagnostic groups. However, possible associations of SZ-PRS with cognition were analyzed in the trans-diagnostic samples rather than separately for the respective diagnostic groups. But there is one exception: Whalley et al. ${ }^{[34]}$ investigated the association between SZ-PRS and some specific cognitive functions exclusively in individuals with MDD. Even though the results did not reveal any significant association, this might be an interesting new approach in the future, in order to further investigate the affective-to-psychotic continuum regarding the effects of genetics on cognitive symptoms ${ }^{[0,10]}$.

\subsection{What are the main limitations of the studies and how can we improve future studies?}

The reviewed studies suggest that the main limiting factor is the lack of statistical power due to insufficient sample size. As more national registries and biobanks provide data on cognitive performance, sufficient sample sizes will become more and more accessible.

Another important limiting factor is the great heterogeneity for measuring cognition, in terms of both the tests used for calculating a factor for general cognitive abilities and tests for specific domains. Furthermore, for genetic studies, there is a lack of studies which replicate findings with the same cognitive battery. The development of the Measurement and Treatment Research to Improve Cognition in Schizophrenia (MATRICS) Consensus Cognitive Battery (MCCB) is an important step in the direction to more consistency within the measures. As it can be seen in the results, the study by Nakahara et al. ${ }^{[27]}$ used the MCCB and revealed significant associations. Therefore, a replication of this study would be worthwhile. 
For future studies it would be desirable to further explore the effects of medications, illicit drug abuse and psychotic symptoms in studies on cognition. Up until now, a gold standard on how to take into account these important confounders of cognitive performance is missing. In order to increase the total sample size for analyses, samples from different studies are often merged. In these cases, it becomes even more crucial to control for potential covariates, since the individuals with psychiatric diseases might not be comparable between the samples. However, controlling for medication, drug abuse or symptoms becomes extra challenging if these variables are assessed in different ways.

Although PRS are in general a good estimate of a person's overall genetic risk for a certain phenotype, there are also some limitations. Firstly, because PRS are based on genome wide association studies (GWAS) findings, they only include information of common variants. Rare variants like copy number variants associated with SZ also explain variability of cognitive performance in controls ${ }^{[52]}$. With the constant advances in sequencing technologies, more information on the role of rare variants will be available and can be considered.

Secondly, the power of a PRS highly depends on the sample size of the discovery GWAS, because bigger discovery samples allow for a more accurate estimation of the effects of the single single-nucleotide polymorphisms (SNPs) ${ }^{[53]}$. Most, but not all studies reported in this review based the calculation of the SZ-PRS on the summary statistics of the second SZ GWAS of the Psychiatric Genomics Consortium (PGC2) GWAS) ${ }^{[25}$. In this GWAS the discovery sample already includes a total of 34,241 cases and 45,604 controls ${ }^{[25]}$, but even larger GWAS would be beneficial.

Thirdly, PRS do not offer an insight into the underlying biological mechanisms influencing cognitive performance. As the effects of SNPs from various pathways are joined to a single score, it should be considered only a very broad measure. If there is prior knowledge of which signaling pathways have an effect on the phenotype, it might be worthwhile to create a PRS that targets those pathways. For example, Rampino and colleagues ${ }^{[45]}$ not only used general SZ-PRS to predict attention, speed of processing and working memory, but also created glutamatergic-PRS. Interestingly, the glutamatergicPRS, but not the general SZ-PRS was significantly associated with attention.

Lastly, PRSs do not include any environmental effects on the phenotype of interest. However, there are important environmental influences on cognitive phenotypes like low socio-economic status, education and nutrition, especially in childhood and adolescence ${ }^{[21]}$.

\subsection{Why is research on this complex topic worthwhile?}

From all we have learned, studying the genetic basis of cognitive deficits in SZ is very time-consuming and expensive. Huge samples are needed with phenotypes, i.e., cognitive tests that are not assessed in routine care. Why could it still be worth the effort?

The idea to use cognitive measures as endophenotypes for SZ is severely challenged by the fact that cognition itself is a complex trait. Nevertheless, as heterogeneous the results are, it remains evident that cognitive impairments come along with SZ as well as other severe psychiatric illnesses. Already the disease itself has a tremendous impact on the life of affected individuals ${ }^{[54]}$. Additionally, they suffer enormously under the cognitive impairments with a strong impact on their quality of life ${ }^{[55-57]}$. Until today there is no psychopharmacological treatment available for cognitive deficits. Furthermore, the degree of cognitive impairments varies greatly between affected individuals, despite being a core feature of SZ. This leads to two big aims of biological psychiatry: the better understanding of the underlying biological processes and the development of treatments specific to the needs of affected individuals. But not only individuals with a psychiatric disease would benefit from a more comprehensive knowledge. Based on the combination of genetic and clinical factors a prediction of cognitive impairments might be possible, with a beneficial impact on both preventive and therapeutic actions. Developing specific training programs to strengthen cognitive performance could increase the resilience of individuals at high risk for SZ. Given the knowledge, that each acute episode is accompanied by a further decrease on social and professional level of the affected individual, costly cognitive trainings could be offered for those with highest needs. Therefore, the research on this topic remains worthwhile.

\section{ACKNOWLEDGEMENTS}

TGS is supported by the Deutsche Forschungsgemeinschaft (DFG) within the framework of the projects www. kfo241.de and www.Psy-course.de (SCHU 1603/4-1, 5-1, 7-1). TGS received additional support from the German Federal Ministry of Education and Research (BMBF) through the Integrated Network IntegraMent (Integrated Understanding of Causes and Mechanisms in Mental Disorders), under the auspices of the e:Med Program (01ZX1614K), and within the framework of the BipoLife network. TGS is also supported by the Dr. Lisa Oehler-Foundation, Kassel (Germany). The funders had no role in study design, data collection and analysis, decision to publish, or preparation of the manuscript. 


\section{CONFLICTS OF INTEREST}

The authors declare that there is no conflict of interest regarding the publication of this paper.

\section{REFERENCES}

1. Fusar-Poli P, Deste G, Smieskova R, Barlati S, Yung AR, Howes $\mathrm{O}$, et al. Cognitive functioning in prodromal psychosis: a meta-analysis. Arch Gen Psychiatry. 2012; 69(6): 562-571.

2. Sitskoorn MM, Aleman A, Ebisch SJH, Appels MCM, Kahn RS. Cognitive deficits in relatives of patients with schizophrenia: a meta-analysis. Schizophr Res. 2004; 71(2-3): 285-295.

3. Hill SK, Reilly JL, Keefe RSE, Gold JM, Bishop JR, Gershon ES, et al. Neuropsychological impairments in schizophrenia and psychotic bipolar disorder: findings from the BipolarSchizophrenia Network on Intermediate Phenotypes (B-SNIP) study. Am J Psychiatry. 2013; 170(11): 1275-1284.

4. Xu G, Lin K, Rao D, Dang Y, Ouyang H, Guo $Y$, et al. Neuropsychological performance in bipolar I, bipolar II and unipolar depression patients: a longitudinal, naturalistic study. J Affect Disord. 2012; 136(3): 328-339.

5. Reichenberg A, Harvey PD, Bowie CR, Mojtabai R, Rabinowitz J, Heaton RK, et al. Neuropsychological function and dysfunction in schizophrenia and psychotic affective disorders. Schizophr Bull. 2009; 35(5): 1022-1029.

6. Szoke A, Meary A, Trandafir A, Bellivier F, Roy I, Schurhoff $F$, et al. Executive deficits in psychotic and bipolar disorders - implications for our understanding of schizoaffective disorder. Eur Psychiatry J Assoc Eur Psychiatr. 2008; 23(1): 20-25.

7. Torrent C, Martínez-Arán A, Amann B, Daban C, Tabarés-Seisdedos R, González-Pinto A, et al. Cognitive impairment in schizoaffective disorder: a comparison with non-psychotic bipolar and healthy subjects. Acta Psychiatr Scand. 2007; 116(6): 453-460.

8. Selzam S, Coleman JRI, Caspi A, Moffitt TE, Plomin R. A polygenic $p$ factor for major psychiatric disorders. Transl Psychiatry. 2018; 8(1): 205.

9. Craddock N, Owen MJ. The Kraepelinian dichotomy-going, going... but still not gone. $\mathrm{Br}$ J Psychiatry. 2010; 196(2): 92-95.

10. Lynham AJ, Hubbard L, Tansey KE, Hamshere $\mathrm{ML}$, Legge SE, Owen MJ, et al. Examining cognition across the bipolar/schizophrenia diagnostic spectrum. J Psychiatry Neurosci JPN. 2018; 43(4): 245-253.

11. Nuechterlein KH, Green MF, Kern RS, Baade LE, Barch DM, Cohen JD, et al. The MATRICS Consensus Cognitive Battery, part 1: test selection, reliability, and validity. Am J Psychiatry. 2008; 165(2): 203-213.

12. August SM, Kiwanuka JN, McMahon RP, Gold JM. The MATRICS Consensus Cognitive Battery (MCCB): clinical and cognitive correlates. Schizophr Res. 2012; 134(1): 76-82.

13. Hilker R, Helenius D, Fagerlund B, Skytthe A, Christensen K, Werge TM, et al. Heritability of Schizophrenia and Schizophrenia Spectrum Based on the Nationwide Danish Twin Register. Biol Psychiatry. 2018; 83(6): 492-498.

14. Pardiñas AF, Holmans P, Pocklington AJ, EscottPrice V, Ripke S, Carrera N, et al. Common schizophrenia alleles are enriched in mutationintolerant genes and in regions under strong background selection. Nat Genet. 2018; 50(3): 381-389.

15. Purcell SM, Wray NR, Stone JL, Visscher PM, O'Donovan MC, Sullivan PF, et al. Common polygenic variation contributes to risk of schizophrenia and bipolar disorder. Nature. 2009; 460(7256), 748-752.

16. Wray NR, Lee SH, Mehta D, Vinkhuyzen AAE, Dudbridge F, Middeldorp CM. Research Review: Polygenic methods and their application to psychiatric traits. J Child Psychol Psychiatry. 2014; 55(10): 1068-1087.

17. Gottesman II, Shields J. Genetic theorizing and schizophrenia. Br J Psychiatry J Ment Sci. 1973; 122(566): 15-30.

18. Gottesman II, Gould TD. The endophenotype concept in psychiatry: etymology and strategic intentions. Am J Psychiatry. 2003; 160(4): 636-645.

19. Deary IJ, Johnson W, Houlihan LM. Genetic foundations of human intelligence. Hum Genet. 2009; 126(1): 215-232.

20. Plomin R, Deary IJ. Genetics and intelligence differences: five special findings. Mol Psychiatry. 2015; 20(1): 98-108.

21. Plomin $R$, von Stumm $S$. The new genetics of intelligence. Nat Rev Genet. 2018; 19(3): 148-159.

22. Savage JE, Jansen $P R$, Stringer $S$, Watanabe $\mathrm{K}$, Bryois J, de Leeuw CA, et al. Genome-wide association meta-analysis in 269,867 individuals identifies new genetic and functional links to intelligence. Nat Genet. 2018; 50(7): 912-919. 
23. Hubbard L, Tansey KE, Rai D, Jones P, Ripke $\mathrm{S}$, Chambert KD, et al. Evidence of Common Genetic Overlap Between Schizophrenia and Cognition. Schizophr Bull. 2016; 42(3): 832-842.

24. Hill WD, Harris SE, Deary IJ. What genome-wide association studies reveal about the association between intelligence and mental health. Curr Opin Psychol. 2018; 27: 25-30.

25. Schizophrenia Working Group of the Psychiatric Genomics Consortium. Biological insights from 108 schizophrenia-associated genetic loci. Nature. 2014; 511(7510): 421-427.

26. Alloza C, Bastin ME, Cox SR, Gibson J, Duff B, Semple SI, et al. Central and non-central networks, cognition, clinical symptoms, and polygenic risk scores in schizophrenia. Hum Brain Mapp. 2017; 38(12): 5919-5130.

27. Nakahara S, Medland S, Turner JA, Calhoun VD, Lim KO, Mueller BA, et al. Polygenic risk score, genome-wide association, and gene set analyses of cognitive domain deficits in schizophrenia. Schizophr Res. 2018 ; 201: 393-399.

28. Ranlund S, Calafato S, Thygesen JH, Lin K, Cahn $\mathrm{W}$, Crespo-Facorro $\mathrm{B}$, et al. A polygenic risk score analysis of psychosis endophenotypes across brain functional, structural, and cognitive domains. Am J Med Genet Part B. 2018; 177(1): 21-34.

29. Shafee R, Nanda P, Padmanabhan JL, Tandon $\mathrm{N}$, Alliey-Rodriguez N, Kalapurakkel S, et al. Polygenic risk for schizophrenia and measured domains of cognition in individuals with psychosis and controls. Transl Psychiatry. 2018; 8(1): 78.

30. van Os J, van der Steen $Y$, Islam MA, Gülöksüz S, Rutten BP, Simons CJ, et al. Evidence that polygenic risk for psychotic disorder is expressed in the domain of neurodevelopment, emotion regulation and attribution of salience. Psychol Med. 2017; 47(14): 2421-2437.

31. Terwisscha van Scheltinga AFT, Bakker SC, van Haren NEM, Derks EM, Buizer-Voskamp JE, Cahn W, et al. Schizophrenia genetic variants are not associated with intelligence. Psychol Med. 2013; 43(12): 2563-70.

32. Walton E, Turner J, Gollub RL, Manoach DS, Yendiki A, Ho B-C, et al. Cumulative genetic risk and prefrontal activity in patients with schizophrenia. Schizophr Bull. 2013; 39(3): 703-711.

33. Wang S-H, Hsiao P-C, Yeh L-L, Liu C-M, Liu C-C, Hwang T-J, et al. Polygenic risk for schizophrenia and neurocognitive performance in patients with schizophrenia. Genes Brain Behav. 2018; 17(1): 49-55.
34. Whalley HC, Adams MJ, Hall LS, Clarke T-K, Fernandez-Pujals AM, Gibson J, et al. Dissection of major depressive disorder using polygenic risk scores for schizophrenia in two independent cohorts. Transl Psychiatry. 2016; 6(11):e938.

35. Xavier RM, Dungan JR, Keefe RSE, Vorderstrasse A. Polygenic signal for symptom dimensions and cognitive performance in patients with chronic schizophrenia. Schizophr Res Cogn. 2018; 12: 11-19.

36. Benca CE, Derringer JL, Corley RP, Young SE, Keller MC, Hewitt JK, et al. Predicting Cognitive Executive Functioning with Polygenic Risk Scores for Psychiatric Disorders. Behav Genet. 2017; 47(1): 11-24.

37. Córdova-Palomera A, Kaufmann T, Bettella F, Wang Y, Doan NT, van der Meer D, et al. Effects of autozygosity and schizophrenia polygenic risk on cognitive and brain developmental trajectories. Eur J Hum Genet EJHG. 2018; 26(7): 1049-1059.

38. Germine L, Robinson EB, Smoller JW, Calkins ME, Moore TM, Hakonarson $\mathrm{H}$, et al. Association between polygenic risk for schizophrenia, neurocognition and social cognition across development. Transl Psychiatry. 2016; 6(10):e924.

39. Hagenaars SP, Harris SE, Davies G, Hill WD, Liewald DCM, Ritchie SJ, et al. Shared genetic aetiology between cognitive functions and physical and mental health in UK Biobank ( $\mathrm{N}=112151)$ and 24 GWAS consortia. Mol Psychiatry. 2016; 21(11): 1624-1632.

40. Hatzimanolis A, Bhatnagar P, Moes A, Wang R, Roussos $\mathrm{P}$, Bitsios $\mathrm{P}$, et al. Common genetic variation and schizophrenia polygenic risk influence neurocognitive performance in young adulthood. Am J Med Genet Part B. 2015; 168B(5): 392-401.

41. Krapohl E, Euesden J, Zabaneh D, Pingault J-B, Rimfeld K, von Stumm S, et al. Phenome-wide analysis of genome-wide polygenic scores. Mol Psychiatry. 2016; 21(9): 1188-1193.

42. Lencz T, Knowles E, Davies G, Guha S, Liewald DC, Starr JM, et al. Molecular genetic evidence for overlap between general cognitive ability and risk for schizophrenia: a report from the Cognitive Genomics consorTium (COGENT). Mol Psychiatry. 2014; 19(2): 168-174.

43. Liebers DT, Pirooznia M, Seiffudin F, Musliner KL, Zandi PP, Goes FS. Polygenic Risk of Schizophrenia and Cognition in a Population-Based Survey of Older Adults. Schizophr Bull. 2016; 42(4): 984-991.

44. Mclntosh AM, Gow A, Luciano M, Davies G, Liewald DC, Harris SE, et al. Polygenic risk 
for schizophrenia is associated with cognitive change between childhood and old age. Biol Psychiatry. 2013; 73(10): 938-943.

45. Rampino A, Taurisano P, Fanelli G, Attrotto M, Torretta S, Antonucci LA, et al. A Polygenic Risk Score of glutamatergic SNPs associated with schizophrenia predicts attentional behavior and related brain activity in healthy humans. Eur Neuropsychopharmacol J Eur Coll Neuropsychopharmacol. 2017; 27(9): 928-939.

46. Riglin L, Collishaw S, Richards A, Thapar AK, Maughan B, O'Donovan MC, et al. Schizophrenia risk alleles and neurodevelopmental outcomes in childhood: a population-based cohort study. Lancet Psychiatry. 2017; 4(1): 57-62.

47. Power RA, Steinberg S, Bjornsdottir G, Rietveld CA, Abdellaoui A, Nivard MM, et al. Polygenic risk scores for schizophrenia and bipolar disorder predict creativity. Nat Neurosci. 2015; 18(7): 953-955.

48. Hill WD, Marioni RE, Maghzian O, Ritchie SJ, Hagenaars SP, Mclntosh AM, et al. A combined analysis of genetically correlated traits identifies 187 loci and a role for neurogenesis and myelination in intelligence. Mol Psychiatry. 2018 Jan 11; doi: 10.1038/s41380-017-0001-5.

49. Sørensen HJ, Debost J-C, Agerbo E, Benros ME, McGrath JJ, Mortensen PB, et al. Polygenic Risk Scores, School Achievement, and Risk for Schizophrenia: A Danish Population-Based Study. Biol Psychiatry. 2018; 84(9): 684-691.

50. Hill WD, Davies G, CHARGE Cognitive Working Group, Liewald DC, Mclntosh AM, Deary IJ. Age-Dependent Pleiotropy Between General Cognitive Function and Major Psychiatric Disorders. Biol Psychiatry. 2016; 80(4): 266-273.
51. Brainstorm Consortium, Anttila V, Bulik-Sullivan B, Finucane HK, Walters RK, Bras J, et al. Analysis of shared heritability in common disorders of the brain. Science. 2018; 360(6395). pii: eaap8757. doi: $10.1126 /$ science.aap8757.

52. Stefansson H, Meyer-Lindenberg A, Steinberg S, Magnusdottir B, Morgen K, Arnarsdottir S, et al. CNVs conferring risk of autism or schizophrenia affect cognition in controls. Nature. 2013; 505(7483): 361-366.

53. Dudbridge F. Power and predictive accuracy of polygenic risk scores. PLoS Genet. 2013; 9(3):e1003348.

54. GBD 2016 Disease and Injury Incidence and Prevalence Collaborators. Global, regional, and national incidence, prevalence, and years lived with disability for 328 diseases and injuries for 195 countries, 1990-2016: a systematic analysis for the Global Burden of Disease Study 2016. Lancet. 2017; 390(10100): 1211-1259.

55. Cruz BF, Resende CB de, Carvalhaes CF, Cardoso CS, Teixeira AL, Keefe RS, et al. Interview-based assessment of cognition is a strong predictor of quality of life in patients with schizophrenia and severe negative symptoms. Rev Bras Psiquiatr Sao Paulo Braz 1999. 2016; 38(3): 216-221.

56. Tas C, Brown E, Cubukcuoglu Z, Aydemir O, Danaci $A E$, Brüne $M$. Towards an integrative approach to understanding quality of life in schizophrenia: the role of neurocognition, social cognition, and psychopathology. Compr Psychiatry. 2013; 54(3): 262-268.

57. Savilla K, Kettler L, Galletly C. Relationships between cognitive deficits, symptoms and quality of life in schizophrenia. Aust N Z J Psychiatry. 2008; 42(6): 496-504. 